\title{
Management of elderly patients with glioblastoma: current status with a focus on the post-operative radiation therapy
}

\author{
Suely Maymone de Melo ${ }^{1}$, Gustavo Nader Marta ${ }^{2,3} \wedge$, Michael Yan ${ }^{4}$, Carol Cruz ${ }^{5}$, Fabio Y. Moraes ${ }^{6}$, \\ Rachel Riera ${ }^{5,7}$
}

${ }^{1}$ Post-graduation Program of Evidence-based Health, Universidade Federal de São Paulo, São Paulo, Brazil; ${ }^{2}$ Department of Radiation Oncology, Hospital Sírio-Libanês, São Paulo, Brazil; ${ }^{3}$ Department of Radiology and Oncology, Radiation Oncology Unit of University of São Paulo, Instituto do Câncer do Estado de São Paulo (ICESP), Faculdade de Medicina da Universidade de São Paulo, São Paulo, Brazil; ${ }^{4}$ Department of Oncology, Division of Radiation Oncology, Queen's University - Kingston Health Science Centre, Kingston, ON, Canada; ${ }^{5}$ Discipline of Evidence-Based Medicine, Universidade Federal de São Paulo - Escola Paulista de Medicina (UNIFESP-EPM), São Paulo, Brazil; ${ }^{6}$ Department of Oncology, Division of Radiation Oncology, Queen's University - Kingston Health Science Centre, Kingston, ON, Canada; ${ }^{7}$ Center of Health Technological Assessment - Hospital Sírio-Libanês, Sao Paulo, Brazil

Contributions: (I) Conception and design: All authors; (II) Administrative support: SM de Melo, GN Marta; (III) Provision of study materials or patients: SM de Melo; (IV) Collection and assembly of data: SM de Melo, GN Marta, R Riera; (V) Data analysis and interpretation: All authors; (VI) Manuscript writing: All authors; (VII) Final approval of manuscript: All authors.

Correspondence to: Gustavo Nader Marta. Department of Radiation Oncology - Hospital Sírio-Libanês, Rua Dona Adma Jafet, 91, Sao Paulo 01308050, SP, Brazil. Email: gustavo.marta@hc.fm.usp.br.

\begin{abstract}
Glioblastoma $(\mathrm{GBM})$ is one of the most malignant primary intracranial neoplasms. This review aims to summarize the treatment of elderly patients with newly diagnosed GBM, with a focus on the radiation therapy (RT) approach. The available literature was reviewed, and we describe the most significant results relating to the post-operative approach of elderly GBM patients. Age limitations in randomized phase III studies have restricted the inclusion of elderly patients, and consequently, limited the generalizability of their results to this patient subset. Chronological age should not prohibit the best treatment, but instead, treatment decisions should consider patient functional status. RT showed efficacy and safety in the elderly population, without compromising quality of life. Hypofractionated RT is not inferior to standard RT. Reduction of overall RT schedule length mitigates the difficulties faced by elderly patients, improving treatment adherence. The addition of both concomitant and adjuvant temozolomide to standard RT is superior to either modality alone and should be the treatment of choice in the subset of patients with good/very good prognosis. It is reasonable to offer hypofractionated RT or temozolomide alone for poor prognosis, and best supportive care (BSC) for very poor prognosis elderly GBM patients. Although combined modality treatment is well established for the management of the good prognosis population, different RT schemes require further investigation with randomized controlled trials to determine the best regimen. A robust analysis of the molecular signatures of GBM in elderly patients might reveal opportunities for clinical protocol modifications to customize management in this group of patients.
\end{abstract}

Keywords: Glioblastoma (GBM); radiation therapy (RT); chemotherapy; temozolomide

Submitted Mar 28, 2020. Accepted for publication Jun 10, 2020.

doi: 10.21037/apm-20-768

View this article at: http://dx.doi.org/10.21037/apm-20-768

$\wedge$ ORCID: 0000-0002-3298-3684. 


\section{Introduction}

Glioblastoma (GBM) is almost universally lethal within two years from diagnosis despite optimal surgical and adjuvant management. It is the most common primary brain tumor (47.7\%) and accounts for $56.6 \%$ of all gliomas. In elderly patients, the annual incidence rate is 3.7 per 100,000 persons (1).

GBM treatment consists of maximum safe resection followed by concomitant radiation therapy (RT) with temozolomide, and then adjuvant temozolomide for six months to one year (2). Median overall survival (OS) is about 16 months for all-comers (3). In some countries, when it is possible, the use of tumor treating fields (TTFields) provides an additional four months benefit for both OS and progression-free survival (PFS) (4).

The definition of elderly is not well established in GBM. It ranges from 60 to 70 years, with most publications setting the lower limit at 65 years $(5,6)$. Elderly GBM patients typically have additional co-morbidities and have an overall worse prognosis (7). For this reason, they tend to receive less aggressive treatment and are traditionally excluded from clinical trials. Recent attempts to offer more aggressive therapy to elderly patients have resulted in improvements in survival, without compromising quality of life (8). Recent trends have moved towards considering management paradigms based more on functional status rather than chronological age (9-11). This review aims to summarize the treatment of elderly patients with newly diagnosed GBM, with a focus on the post-operative RT approach.

\section{Methods}

A systematic review in accordance with The Cochrane Collaboration Handbook of Interventions Systematic Reviews was performed (12). The electronic literature searches without any language restrictions or publication year was conducted in 3 different electronic databases: MEDLINE (1966 to 28 February 2020, via PubMed), EMBASE (1988 to 27 February 2020, via Elsevier) and Cochrane Central Register of Controlled Trials (CENTRAL, 2020 issue 7, via Wiley). We used the following terms and search strategies: (Glioblastomas OR glioma, Grade IV OR Glioblastoma Multiforme OR Giant Cell Glioblastoma OR Giant Cell Glioblastomas OR Glioblastoma, Giant Cell OR Glioblastomas, Giant Cell) AND (Radiotherapy OR Radiotherapies OR Radiation Therapy OR Radiation Therapies OR Therapies, Radiation
OR Therapy, Radiation OR Radiation Treatment OR Radiation Treatments OR Treatment, Radiation OR Radiotherapy, Targeted OR Radiotherapies, Targeted OR Targeted Radiotherapies OR Targeted Radiotherapy OR Targeted Radiation Therapy OR Radiation Therapies, Targeted OR Targeted Radiation Therapies OR Therapies, Targeted Radiation OR Therapy, Targeted Radiation OR Radiation Therapy, Targeted) AND (Aged OR Elderly). We screened systematic reviews, retrospective studies, and prospective trials for material to incorporate within the discussion. An independent review of the references was performed. We selected 42 manuscripts for analysis that most addressed our study question (Figure 1).

\section{Results}

\section{To treat or not to treat}

In clinical practice, the decision to treat or not to elderly GBM patients have been assessed by several studies.

Kita $e t a l$. evaluated a cohort of 715 GBM patients from 1980 to 1994 in Zurich, Switzerland. Among patients treated with surgery and RT or RT alone, younger age ( $<60$ versus $\geq 60$ years) was significantly associated with higher OS. This association was not evident in patients treated with surgery alone or best supportive care (BSC). These results suggest that although there is benefit of treatment at all ages, it is more pronounced in the younger population. However, amongst patients $<65$ years of age, $82 \%$ of patients underwent surgery and/or RT, while only $47 \%$ of patients aged $\geq 65$ years and $25 \%$ of patients $\geq 75$ years received these treatments. In the population $\geq 60$ years, there was no difference in OS between the age subgroups. Using the age range of 60-64 years as the reference, the hazard ratio (HR) for OS was 1.11 for patients 65-69 [95\% confidence interval (CI), 0.84-1.48]; 0.98 (95\% CI, 0.72-1.34) for patients 70-74; and 1.05 (95\% CI, 0.76-1.46) for patients $\geq 75$ years of age (13).

A population-based study using the Surveillance, Epidemiology, and End Results (SEER) database, compared different types of treatment in 2,836 GBM patients aged $\geq 70$ years. Benefit in OS was observed in patients treated with surgery and/or RT over those who received BSC. The median OS was 8 months for the patients treated with surgery and RT, 4 months for RT only group, and 3 months for the surgery only group. Patients receiving BSC had a median OS of only 2 months. In all patients who received RT, the multivariate analysis showed a significant benefit in 


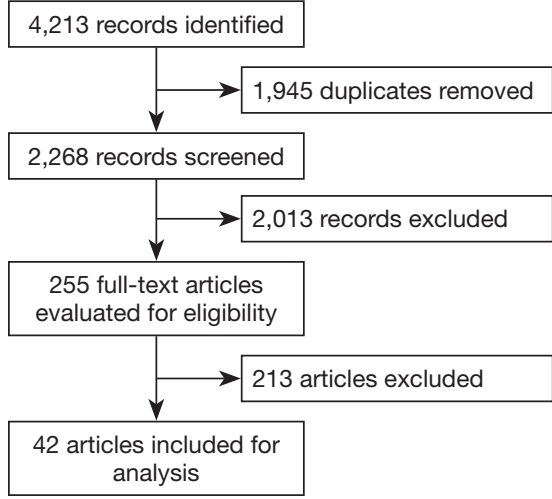

Figure 1 PRISMA flow diagram.

OS (HR $=0.43 ; 95 \%$ CI, 0.38-0.49) compared to those who $\operatorname{did}$ not (8).

Harris et al., retrospectively evaluated 108 GBM patients aged $\geq 75$ years, treated with intensity modulated radiotherapy (IMRT), from 2006 to 2016. The median age was 78.7 years, with $38.4 \%$ of patients aged $\geq 80$ years. The dose ranged from $<40$ Gy (hypo-fractionated RT, HRT) to 60 Gy (standard RT, sRT). Forty patients received temozolamide (TMZ), of which 39 were received concurrent IMRT. The OS for the entire cohort was 6.7 months (95\% CI, 4.7-9.1). For patients receiving BSC, RT alone, and RT+TMZ, the OS was 1.9, 6.3, and 13.2 months respectively, with a significant difference between the groups. The dose of IMRT had no impact on OS. Patients aged 74-80 years had a median OS of 11.5 months (95\% CI, 7.1-13.2), while those $\geq 80$ years old had a median OS of 9.3 months (95\% CI, 4.3-10.6). This study may have been limited by selection bias, since patients with better prognosis received more aggressive treatment, as well as its small study size (14).

Mak et al., using data from the National Cancer Data Base (NCDB), compared HRT $(n=304)$ versus sRT $(n=4,294)$ in $G B M$ patients aged $\geq 70$ years. They defined HRT as 34-42 Gy in 2.5-3.4 Gy/fraction and sRT as 58-63 Gy in 1.8-2.0 Gy/fraction. The median OS was 4.9 months for the HRT group and 8.9 months for the sRT group (HR 1.51; $95 \%$ CI, $1.33-1.73, \mathrm{P}=0.0001)$. The OS for the HRT and sRT groups respectively were $13.2 \%$ versus $34.7 \%$ at one year, $5.1 \%$ versus $5.4 \%$ at two years, and $1.8 \%$ versus $5.4 \%$ at three years. Even after adjusting for prognostic factors, HRT resulted in a lower OS. Consideration should be made about selection bias, because patients in the HRT group were older, had worse co-morbidities, and were less likely to undergo more aggressive resection or chemotherapy. In addition, Karnofsky Performance Status (KPS) was only available for $3.1 \%$ of patients, and there was a great deal of missing data regarding the molecular characteristics of the tumors (15).

Bingham et al., in a retrospective analysis of elderly patients with GBM using the NCDB [2005-2012], also observed a benefit of sRT over HRT in terms of OS. However, when they excluded patients who died within 90 days in order to reduce the bias of worse prognosis patients receiving HRT, this difference disappeared (16).

\section{Randomized control trials (RCT)}

\section{Elderly patients treated with standard RT}

Stupp et al. evaluated 573 adult patients (18-70 years old) with GBM in a phase III RCT (EORTC/NCIC). All patients were WHO performance status (WHO PS) $0-2$. In the experimental arm, patients were treated with concomitant sRT and TMZ $\left(75 \mathrm{mg} / \mathrm{m}^{2} /\right.$ day) followed by adjuvant $(200 \mathrm{mg} / \mathrm{d}, 5 / 28$ days $)$ for six months, compared to sRT alone in the control. They observed a significant increase in OS (14.6 versus 12.1 months) in the experimental arm; this became standard treatment. In the subgroup analysis of the elderly population ( $\geq 60$ years), they observed a slight increase in OS in the combined treatment arm (HR 0.7; 95\% CI, 0.50-0.97). This difference increased after two (21.8 versus 5.7 ), three (12.3 versus 2.3 ), four (8.8 versus 2.3 ) and five years (6.6 versus 0 ) of follow-up. However, due to the small number of patients $(n=170)$, the interaction test was not significant, and the power was not sufficient to allow for definitive conclusions (3).

Keime-Guibert et al. compared postoperative RT (50.4 Gy in 1.8 Gy per fraction for a total of 28 fractions) versus BSC in GBM patients aged $\geq 70$ years and with KPS $\geq 70$. The trial was closed after interim analysis, because it reached the pre-established efficacy limit for superiority in the RT group, with an increase in median OS from 16.9 to 29.1 weeks ( $\mathrm{HR}=0.47 ; 95 \% \mathrm{CI}, 0.29-0.76, \mathrm{P}=0.002$ ) without compromise of quality of life (QoL). They did not observe severe adverse events (AE) attributed to the RT (17).

Wick et al. (NOA-08) compared TMZ alone $\left(100 \mathrm{mg} / \mathrm{m}^{2}\right.$ per day, in alternate weeks) versus sRT in patients with GBM $(89 \%)$ or astrocytoma grade III, aged $\geq 60$ years and KPS $\geq 60$, in a randomized, non-inferiority trial. The median OS was 8.6 months (95\% CI, 7.3-10.2) in the TMZ group and 9.6 months (95\% CI, 8.2-10.8) in the 
RT group (HR 1.09; 95\% CI, 0.84-1.42, $\mathrm{P}=0.03$ ). The PFS was 3.3 months (95\% CI, 3.2-4.1) in the TMZ group and 4.7 months $(4.2-5.2)$ in the RT group (HR 1.15; 95\% CI, 0.92-1.43, P=0.04). O-6-methylguanine-DNA methyltransferase (MGMT) status was available in 209 of the $412(51 \%)$ randomized patients. PFS was higher in the TMZ arm in MGMT mutated patients (8.4 versus 4.6 months), whereas in the MGMT wildtype population, PFS was significantly higher in the RT arm (4.6 versus 3.3 months). They concluded that TMZ alone was not inferior to RT. The toxicity was higher in the TMZ arm. MGMT status was considered predictive for TMZ response. QoL was assessed in $82 \%$ of the participants, and was similar in the two groups (18).

\section{Elderly patients treated with bypofractionated RT}

Bleehen et al. (Medical Research Council trial: MRC BR2) compared HRT (45 Gy in 20 fractions over 4 weeks) versus sRT, in 474 patients with malignant glioma (astrocytoma GIII and GBM). They observed a modest, but significant increase in median OS in the sRT arm (from 9 to 12 months). However, in the elderly and/or poor prognosis population, they did not find any significant difference between the two groups. No significant acute toxicity was observed in either arm, and late toxicity was not assessed (19).

Roa et al. evaluated two RT schemes $(60 \mathrm{~Gy} / 30$ fractions versus $40 \mathrm{~Gy} / 15$ fractions) in patients $\geq 60$ years and KPS $\geq 50$ in an equivalency trial. There was no significant difference in median OS between the two groups (5.1 versus 5.6 months, respectively, $\mathrm{P}=0.57$ ). Fewer patients in the HRT arm stopped before completion of treatment (10\% versus $25 \%$ ). The KPS score was similar between patients in the two schemes and the corticosteroids dependence was lower in the HRT arm. They intended to assign 224 patients, but in October 2001, the steering committee decided to close the study after recruiting 100 patients. This was due to the fact that to exclude a small difference in survival, the required number of patients would not be feasible (20).

Malmström et al. (NORDIC study) investigated patients with GBM, aged $\geq 60$ and Eastern Cooperative Oncology Grouop (ECOG) 0-2. They randomized the patients to three arms: two arms on different schemes of RT (sRT versus HRT) and one arm on TMZ alone. Patients in the sRT arm received 60 Gy in 30 fractions over six weeks, while those on the HRT arm received 34 Gy in 10 fractions over two weeks. Patients in the TMZ arm received $200 \mathrm{mg} / \mathrm{m}^{2} /$ day for five days, every 28 days for 6 cycles. No significant difference was observed between the two radiotherapy regimens in patients aged 65 to 70 years. In the subgroup of patients $\geq 70$ years, the median OS was significantly higher in the HRT arm when compared to sRT (7.0 versus 5.2 months, $\mathrm{P}=0.02$ ), and rates of completion were higher as well (94.9\% versus $72 \%)$. This higher rate likely contributed to the increase in survival in that group. The TMZ arm was not superior to HRT, but like HRT, this group had a better OS than the sRT arm (21).

Roa 2015/Castro 2017 [The International Atomic Energy Agency (IAEA)] randomized 98 elderly and/or frail (KPS 50-70\%) patients with newly diagnosed GBM to receiving $25 \mathrm{~Gy} / 5$ fractions over 1 week in arm one versus $40 \mathrm{~Gy} / 15$ fractions over 3 weeks in arm two. The primary end point was OS, and a non-inferiority margin of $15 \%$ was proposed. In a post-hoc analysis (Castro, 2017), the authors evaluated only the subgroup of 61 elderly patients ( $\geq 65$ years, KPS 50-90). There was no significant difference in median OS; it was 6.8 months $(95 \%$ CI, 4.5-9.1) in arm one versus 6.2 months (95\% CI, 4.7-7.7) in arm two (HR $=1.003 ; 95 \%$ CI, 0.660-1.524; $\mathrm{P}=0.936$ ). Similarly, no significant difference in PFS was observed, with 4.3 months (95\% CI, 2.6-5.9) in arm one versus 3.2 months (95\% CI, 1-6.3) in arm two $(\mathrm{P}=0.706)$. QoL was measured by EORTC questionnaires - QLQ-C30 and QLQ-BN20 in all study patients, and no difference was observed between the groups. There was no grade 3 acute toxicity. The limitations in this study were the small number of patients evaluated, and that it was a post-hoc analysis (22-24).

The NCIC/EORTC study evaluated patients aged $\geq 65$ and randomized them to receive HRT (40 Gy in 15 fractions) with concomitant TMZ $\left(75 \mathrm{mg} / \mathrm{m}^{2}\right.$ per day) and adjuvant TMZ (150-200 mg/m $\mathrm{m}^{2}$ every 5 days, each cycle of 28 days/12 cycles or until progression) versus HRT alone. There was an improvement in OS and PFS in the combined modality arm (median OS 9.3 versus 7.6 months, $\mathrm{HR}=0.67, \mathrm{P}<0.0001$ and PFS 5.3 versus 3.9 months, HR $=0.50 ; \mathrm{P}<0.001)$. The greatest $\mathrm{OS}$ benefit was observed in patients with tumors with MGMT promoter methylation (13 months). The adverse effects of nausea, vomiting and constipation were higher in the combined modality arm. The authors did not observe any difference in resection length, ECOG status, QoL, or mental status, when stratifying by age range (10). This is currently the standard treatment of elderly, newly diagnosed GBM patients who 
are unfit for conventional treatment (Stupp regimen). The latter should be considered in patients $65-70$ years old, with a good/very good prognosis. In patients who do not tolerate the combination therapy, RT or TMZ alone (based on MGMT) may be used.

The results of these RCTS are summarized in Table 1.

\section{Discussion}

Primary GBM is a disease predominant in the elderly, although there is a paucity of evidence to support the treatment approach for this population (1).

Age is a well-known negative prognostic factor in adult GBM patients (25). Age limitations in randomized phase III studies have restricted their inclusion and, consequently, limited the generalizability of study results to this subset of patients. Moreover, medical comorbidity and poor functional reserve that are frequently observed in elderly patients support the development of differential treatment recommendations (26). Elderly patients with GBM frequently do not undergo the standard treatment. A tendency of abridged treatment is seen, unlike in younger patients who are often treated more aggressively. Thus, understanding the influence of age in this population is very important in clinical decision-making.

The two cohort studies evaluated in this review showed an OS benefit of treatment (surgery and RT or RT alone) over BSC, at any age although more pronounced in younger patients ( $<60$ versus $\geq 60$ years) $(13,26)$. Interesting, when Kita et al. compared different age ranges in the population over 60 years of age, there was no difference in OS, suggesting a homogeneous response to treatment in this population. In a recent retrospective study with 66 elderly GBM patients treated with sRT or dose-escalated RT (range, 60-81 Gy) and TMZ, a comparison of patients age $\geq 70$ (71-81 years) to those who were $60-70$, the OS was similar between the two groups after bias adjustment for bias (age $\geq 70$ were less likely to receive adjuvant TMZ; $\mathrm{P}=0.02$ ) (27). In the phase III trial by Stupp et al., in patients over 65 years old, the baseline factors that correlated with OS were surgery and Mini-Mental State Examination (MMSE) score (3). Age was found to be inversely correlated with OS in the combination treatment arm $(8.7 \mathrm{~m}$ for age $\leq 70$ years and $10 \mathrm{~m}$ for $>70$ years); although this suggests a selection bias (patients with age $\leq 70$ years in good KPS probably were treated by the Stupp regimen).

In a RCT comparing sRT versus BSC for elderly patients, the efficacy of sRT, was demonstrated without compromise of QoL, severe adverse effects, or cognition (17). Harris et al. used IMRT to patients aged $\geq 75$ years (about $40 \%$ aged $\geq 80$ years), with a significant benefit in the RT group, over BSC (14). Chronological age should not be a limitation in choosing the best treatment; the decision should centre on functional status. Based on these studies, radiotherapy was found to be effective and safe in the elderly population.

When comparing sRT versus chemotherapy (TMZ), Wick et al. found no difference in OS (18). For PFS, sRT was superior to TMZ in the MGMT wildtype group. The opposite was true in the MGMT mutated group. The incidence of AEs was greater in the chemotherapy arm.

HRT was compared with sRT in two cohorts evaluated in this review $(15,16)$. Both found superior OS in the sRT group, although the first study recognized selection bias in favor of sRT group. HRT patients had worse comorbidities, and were less likely to undergo more aggressive resection or chemotherapy. In addition, there was a paucity of KPS data (only $3 \%$ of patients), which is a known, significant prognostic factor. Bingham et al., after reducing the bias of choosing the HRT regimen for patients with worse prognosis, did not find any difference in OS between both treatments (16).

Four RCTs evaluated HRT. Three trials (19-21) that performed a direct comparison of HRT versus sRT showed similar survival, except for the group over 70 years of age (21), for which hypo-fractionation was superior (HR $=0.59 ; 95 \% \mathrm{CI}, 0.37-0.93, \mathrm{P}=0.02)$. This may have been attributed to better adherence to the assigned therapy in the HRT group (19). The fourth trial (24) evaluated two hypofractionation schedules, with no difference in survival between them. In this study, the elderly patients were analyzed, post-hoc, as a subset of a larger population of elderly and/or frail patients. QoL was also not significantly different between groups. In the HRT arm (22), fewer patients stopped treatment prematurely. Reduction in the length of RT appears to improve the treatment adherence of elderly patients (21).

The addition of TMZ to sRT resulted in superior OS when compared with RT or TMZ alone in observational studies $(11,28)$ and in two RCTs. In RCTs, Stupp et al., in the subgroup analysis of the elderly population, found a benefit with the association of TMZ to sRT. The caveat however, was that this observation was not adequately powered due to small number of patients (3). A recent trial randomizing patients to HRT versus HRT with concomitant and adjuvant temozolomide demonstrated the 


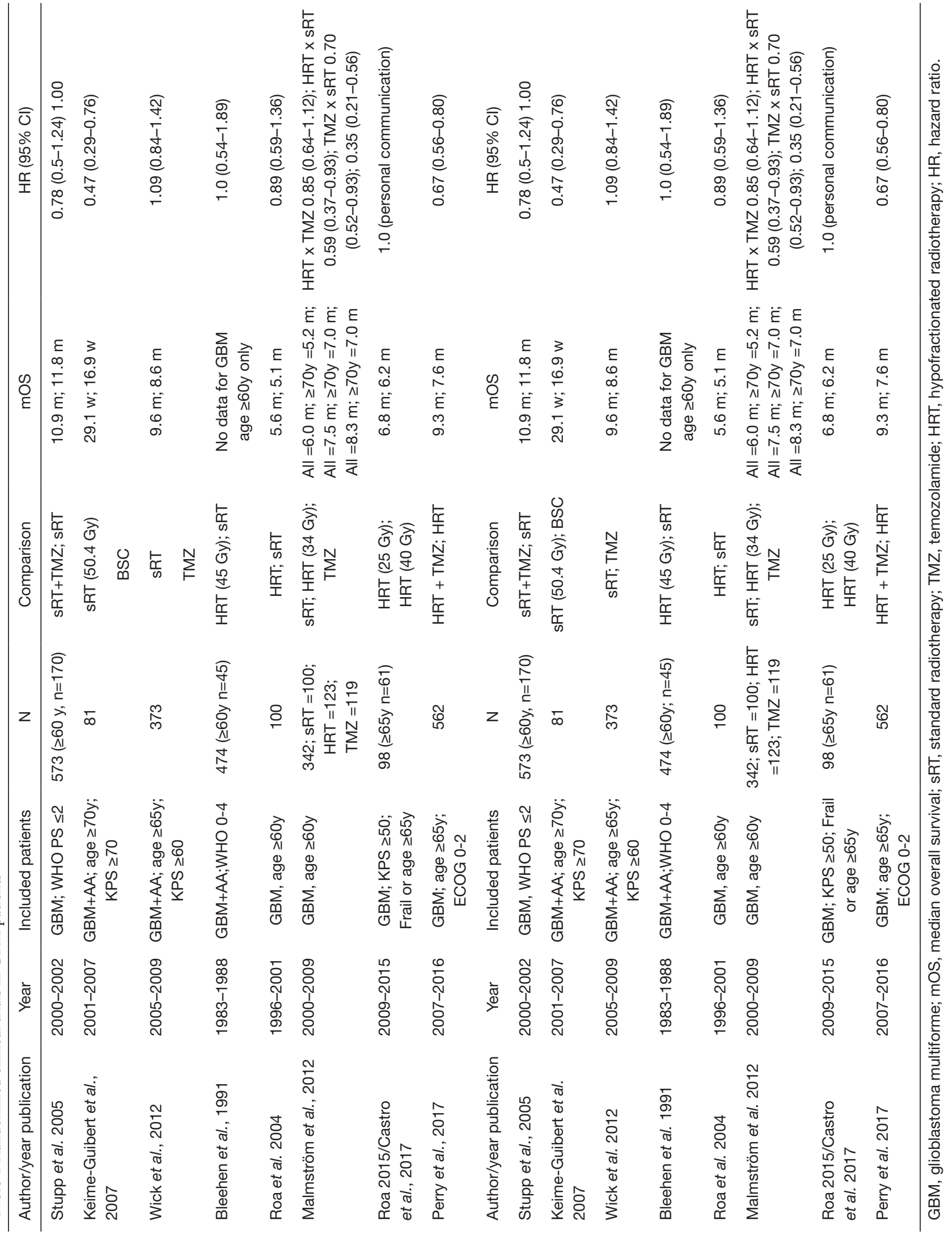




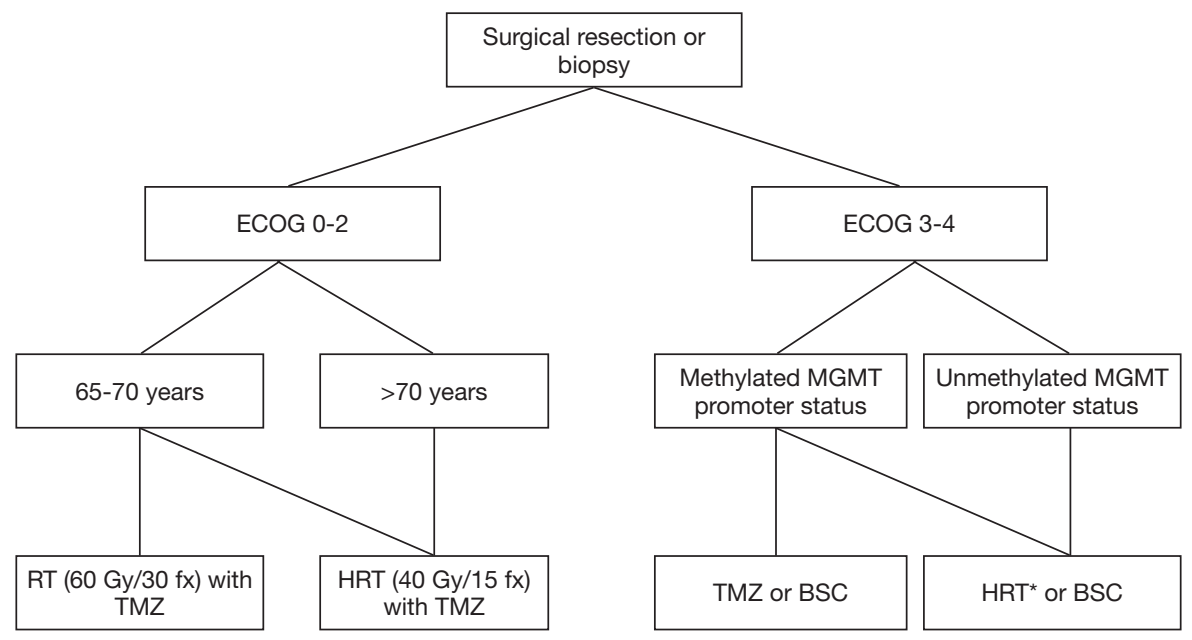

Figure 2 Flow diagram of treatment: elderly GBM patients. *, consider 25 Gy/5 fractions. BSC, best supportive care; ECOG, Eastern Cooperative Oncology Group Performance Status; HRT, hypofractionated radiation therapy; MGMT, O-6-methylguanine-DNA methyltransferase; RT, radiation therapy; TMZ, temozolomide.

superiority of the combined regimen with a median OS of 9.3 versus 7.6 months in the HRT alone arm (10).

The addition of TMZ to RT should be the treatment of choice in good/very good prognosis patients. Monotherapy (RT or TMZ) and BSC should be considered in those with poor performance status, which was not evaluated in the Perry trial (WHO PS 0-2) (10). Although combined modality treatment is well established for the management of good prognosis patients, these RT schemes have to be further investigated with RCTs to determine the best regimen.

It is critical that future studies investigate other treatment approaches to guide the development of guidelines that allow for the reduction of toxicity. Hence, further RCTs must integrate clinical endpoints to evaluate the treatmentrelated toxicities of different therapeutic modalities and possible consequences on QoL. Elderly GBM patients may be categorized by a different genetic signature, which has yet to be described. Investigating the molecular biology of GBM in elderly patients might expose opportunities for modifying clinical protocols to customize care in this group of patients (29).

In Figure 2, we present a flow diagram to support clinical decision making when treating elderly GBM patients.

\section{Conclusions}

Randomized control trial evidence has shown that, for elderly patients with GBM, surgery followed by RT is superior to BSC. The addition of TMZ to RT in the concomitant and adjuvant setting increased OS and PFS over each monotherapy alone, and now is considered for good prognosis patients. It is reasonable to offer hypofractionated RT, chemotherapy alone, or BSC for poor prognosis patients.

\section{Acknowledgments}

Funding: This research did not receive any specific grant from funding agencies in the public, commercial, or notfor-profit sectors.

\section{Footnote}

Peer Review File: Available at http://dx.doi.org/10.21037/ apm-20-768

Conflicts of Interest: All authors have completed the ICMJE uniform disclosure form (available at http://dx.doi. org/10.21037/apm-20-768). The authors have no conflicts of interest to declare.

Ethical Statement: The authors are accountable for all aspects of the work in ensuring that questions related to the accuracy or integrity of any part of the work are appropriately investigated and resolved.

Open Access Statement: This is an Open Access article 
distributed in accordance with the Creative Commons Attribution-NonCommercial-NoDerivs 4.0 International License (CC BY-NC-ND 4.0), which permits the noncommercial replication and distribution of the article with the strict proviso that no changes or edits are made and the original work is properly cited (including links to both the formal publication through the relevant DOI and the license). See: https://creativecommons.org/licenses/by-nc-nd/4.0/.

\section{References}

1. Ostrom QT, Gittleman H, Liao P, et al. CBTRUS Statistical Report: Primary brain and other central nervous system tumors diagnosed in the United States in 20102014. Neuro Oncol 2017;19:v1-88.

2. Stupp R, Mason WP, van den Bent MJ, et al. Radiotherapy plus concomitant and adjuvant temozolomide for glioblastoma. N Engl J Med 2005;352:987-96.

3. Stupp R, Hegi ME, Mason WP, et al. Effects of radiotherapy with concomitant and adjuvant temozolomide versus radiotherapy alone on survival in glioblastoma in a randomised phase III study: 5-year analysis of the EORTC-NCIC trial. Lancet Oncol 2009;10:459-66.

4. Stupp R, Taillibert S, Kanner A, et al. Effect of TumorTreating Fields Plus Maintenance Temozolomide vs Maintenance Temozolomide Alone on Survival in Patients With Glioblastoma: A Randomized Clinical Trial. JAMA 2017;318:2306-16.

5. Lewis JH, Kilgore ML, Goldman DP, et al. Participation of patients 65 years of age or older in cancer clinical trials. J Clin Oncol 2003;21:1383-9.

6. Rampling R, Erridge S. Management of central nervous system tumours in the elderly. Clin Oncol (R Coll Radiol) 2014;26:431-7.

7. Hoffe S, Balducci L. Cancer and age: general considerations. Clin Geriatr Med 2012;28:1-18.

8. Scott JG, Bauchet L, Fraum TJ, et al. Recursive partitioning analysis of prognostic factors for glioblastoma patients aged 70 years or older. Cancer 2012;118:5595-600.

9. Lawrence YR, Wang M, Dicker AP, et al. Early toxicity predicts long-term survival in high-grade glioma. $\mathrm{Br} \mathrm{J}$ Cancer 2011;104:1365-71.

10. Perry JR, Laperriere N, O'Callaghan CJ, et al. ShortCourse Radiation plus Temozolomide in Elderly Patients with Glioblastoma. N Engl J Med 2017;376:1027-37.

11. Rusthoven CG, Koshy M, Sher DJ, et al. CombinedModality Therapy With Radiation and Chemotherapy for
Elderly Patients With Glioblastoma in the Temozolomide Era: A National Cancer Database Analysis. JAMA Neurol 2016;73:821-8.

12. Higgins JPT GSe. Cochrane Handbook for Systematic Reviews of Interventions: version 5.0.2 [updated September 2011]. The Cochrane Collaboration 2008. 2011.

13. Kita D, Ciernik IF, Vaccarella S, et al. Age as a predictive factor in glioblastomas: population-based study. Neuroepidemiology 2009;33:17-22.

14. Harris G, Jayamanne D, Wheeler H, et al. Survival Outcomes of Elderly Patients With Glioblastoma Multiforme in Their 75th Year or Older Treated With Adjuvant Therapy. Int J Radiat Oncol Biol Phys 2017;98:802-10.

15. Mak KS, Agarwal A, Qureshi MM, et al. Hypofractionated short-course radiotherapy in elderly patients with glioblastoma multiforme: an analysis of the National Cancer Database. Cancer Med 2017;6:1192-200.

16. Bingham B, Patel CG, Shinohara ET, et al. Utilization of hypofractionated radiotherapy in treatment of glioblastoma multiforme in elderly patients not receiving adjuvant chemoradiotherapy: A National Cancer Database Analysis. J Neurooncol 2018;136:385-94.

17. Keime-Guibert F, Chinot O, Taillandier L, et al. Radiotherapy for glioblastoma in the elderly. N Engl J Med 2007;356:1527-35.

18. Wick W, Platten M, Meisner C, et al. Temozolomide chemotherapy alone versus radiotherapy alone for malignant astrocytoma in the elderly: the NOA-08 randomised, phase 3 trial. Lancet Oncol 2012;13:707-15.

19. Bleehen NM, Stenning SP. A Medical Research Council trial of two radiotherapy doses in the treatment of grades 3 and 4 astrocytoma. The Medical Research Council Brain Tumour Working Party. Br J Cancer 1991;64:769-74.

20. Roa W, Brasher PM, Bauman G, et al. Abbreviated course of radiation therapy in older patients with glioblastoma multiforme: a prospective randomized clinical trial. J Clin Oncol 2004;22:1583-8.

21. Malmstrom A, Gronberg BH, Marosi C, et al.

Temozolomide versus standard 6-week radiotherapy versus hypofractionated radiotherapy in patients older than 60 years with glioblastoma: the Nordic randomised, phase 3 trial. Lancet Oncol 2012;13:916-26.

22. Roa W, Kepka L, Kumar N, et al. International Atomic Energy Agency Randomized Phase III Study of Radiation Therapy in Elderly and/or Frail Patients With Newly Diagnosed Glioblastoma Multiforme. J Clin Oncol 
2015;33:4145-50.

23. Roa W, Ghosh S, Zhu Q, et al. Reply to H. Kim et al and T. Finazzi. J Clin Oncol 2016;34:2192-3.

24. Guedes de Castro D, Matiello J, Roa W, et al. Survival Outcomes With Short-Course Radiation Therapy in Elderly Patients With Glioblastoma: Data From a Randomized Phase 3 Trial. Int J Radiat Oncol Biol Phys 2017;98:931-8.

25. Stummer W, Nestler U, Stockhammer F, et al. Favorable outcome in the elderly cohort treated by concomitant temozolomide radiochemotherapy in a multicentric phase II safety study of 5-ALA. J Neurooncol 2011;103:361-70.

26. Scott JG, Suh JH, Elson P, et al. Aggressive treatment is appropriate for glioblastoma multiforme patients 70 years

Cite this article as: de Melo SM, Marta GN, Yan M, Cruz C, Moraes FY, Riera R. Management of elderly patients with glioblastoma: current status with a focus on the post-operative radiation therapy. Ann Palliat Med 2020;9(5):3553-3561. doi: 10.21037/apm-20-768 old or older: a retrospective review of 206 cases. Neuro Oncol 2011;13:428-36.

27. Jackson WC, Tsien CI, Junck L, et al. Standard dose and dose-escalated radiation therapy are associated with favorable survival in select elderly patients with newly diagnosed glioblastoma. J Neurooncol 2018;138:155-62.

28. Franceschi E, Depenni R, Paccapelo A, et al. Which elderly newly diagnosed glioblastoma patients can benefit from radiotherapy and temozolomide? A PERNO prospective study. J Neurooncol 2016;128:157-62.

29. Hegi ME, Stupp R. Neuro-oncology: in search of molecular markers of glioma in elderly patients. Nat Rev Neurol 2013;9:424-5. 